\title{
Speech therapy for psychogeriatric services
}

\section{Luxury or necessity?}

\author{
Helen Griffiths, Senior Speech Therapist; and Bob Baldwin, Consultant \\ Psychogeriatrician, York House, Manchester Royal Infirmary, Manchester
}

\begin{abstract}
A psychogeriatrician was recently given the opportunity of five sessions of speech therapy for his service. His response was that he had no need of a speech therapist as he was perfectly well able to do his own assessment of dysphasia. This attitude is perhaps understandable since there has been so little exposure to the skills of speech therapists within this clinical field. In 1985 a full-time speech therapist was appointed by Central Manchester Health Authority to be part of the multidisciplinary team working in old age psychiatry. At the time this was, to our knowledge, the first full-time position of its kind. This article reviews progress thus far and outlines the contribution of speech therapy skills to the management of the elderly mentally ill.

Since this new post was a departure from the speech therapist's role in acute and rehabilitative medical settings and since little was known about the practical needs of this group as regards language and communication problems, it was decided to operate an open referral system and examine patterns as they emerged. In addition to offering assessment and advice on management, commitments were also made to the areas of research and teaching.
\end{abstract}

\section{Clinical aspects}

Over the three year period March 1985-88 a total of 280 new referrals were made, with roughly equal numbers in each year: $35 \%$ from the in-patient areas; $35 \%$ from the psychogeriatric day hospital, $25 \%$ directly from the community, and $5 \%$ from other hospital departments. A considerable number have been carried over each year for ongoing work.

Of the new referrals the highest proportion, $75 \%$, were judged by the clinicians to have a chronic organic psychosyndrome; non-organic cases were $25 \%$, of whom $75 \%$ were depressed and $25 \%$ suffering from paranoid states. The reasons for referral differed across the groups but included diagnostic assessment, assessment of communication skills, advice on communication as part of a management plan and management of hearing loss. A small number of individuals were referred for one to one direct treatment.

The following cases illustrate the contribution of speech therapy: the first two concerning issues of assessment; cases three and four regarding management.

\section{Case 1}

Mrs B, aged 63, was referred for speech therapy following what was thought to be a slight CVA. Detailed language assessment revealed a pattern of difficulty not consistent with any of the dysphasic syndromes associated with a focal vascular lesion. Further investigation and long-term follow up has revealed a progressive illness with a fronto-temporal emphasis in which language disturbance was the presenting symptom. The speech therapist's assessment made a valuable contribution in reaching the correct diagnosis, with implications for the treatment plan and prognosis.

Case 2

Miss C, aged 66, was referred to the psychogeriatric services because of a history of self neglect and increasing dependence on her niece. Formal psychometric testing was difficult because of her near echolalic speech. Speech therapy assessment revealed a discrepancy between language competence and performance with a pattern of errors not consistent with the semantic breakdown associated with Alzheimer's Disease (Bayles \& Koszniak, 1987). The poverty of spontaneous utterances, the retention of understanding, and her echolalic speech indicated specific frontal lobe damage. Investigations confirmed that she was suffering from the recently described dementia of the frontal type (Neary et al, 1988). Further identification of which components of language were preserved also suggested ways of improving communication with her carers.

\section{Case 3}

Miss D, aged 76, had been transferred to a residential home for the elderly with a diagnosis of multi-infarct 
dementia. There staff described her as unco-operative and aggressive. She was dysphasic, and her output included many perseverative utterances. Her disordered speech led staff to assume that she could not understand anything. Matters were further exacerbated by significant hearing loss. Assessment revealed that she could understand, provided verbal input was simple and accompanied by appropriate gesture, especially where close personal contact was necessary. Sharing these findings with care staff, along with simple strategies to aid communication, e.g. a visual chart with symbols relevant to activities of daily living, secured her co-operation, eased staff apprehension and improved her quality of life.

\section{Case 4}

Mrs $\mathbf{M}$ is a long-stay resident with dementia in York House whose speech is unintelligible. Careful assessment and observation has revealed a basic level of comprehension which can be supplemented by the use of gesture and physical contact. There is also some preservation of oral reading and rhythmic ability. The former has been used to shape linguistic output during structured sessions and the latter has been enhanced by the use of music. More importantly, the analysis of the problem has enabled staff to maximise their interaction using everyday activities, such as dressing and mealtimes, and to allow Mrs M to express some choice because staff are now sensitive to her nonverbal communication.

Traditionally, patients referred are seen on a one to one basis for speech therapy. Our experience suggests that a very different approach is required within this clinical setting. The emphasis is much more on careful assessment followed by imparting skills and strategies for coping with communication breakdown to other professional and informal carers and relatives. Patients were frequently referred from discussion in team meetings or ward rounds and many useful interventions might have been missed if the speech therapist had not been a team member. Clearly attendance at every ward round would be unrealistic and unnecessary. What seems to have happened is that the consciousness of the rest of the team toward communication problems, not merely dysphasia, has been raised as a result of on-going exposure to the speech therapist and her skills. Presumably the same could occur even if only a few sessions of speech therapy were available provided they were regular and were linked directly to the psychogeriatric multi-disciplinary team. It is doubtful whether the same would apply within a setting where patients are seen 'off site' within a speech therapy department.

In the past, language disturbance has been regarded as a late feature of dementia. In fact, if specifically sought, language difficulties are frequent and occur early in the course of the illness (Bayles, 1982). In addition, the frequent emphasis on 'problem' behaviours within psychogeriatric practice ignores the fact that many carers are as distressed by the loss of meaningful interaction as they are by difficult behaviours (Gilleard, 1984). One of the things most appreciated by the carers of dementia patients in day hospital attendance is seeing their relative engaged in activity and conversation. Not infrequently we hear reports of both increased quality and quantity of conversation for several hours after returning from the day hospital. Thus regular communication groups are now used as an economic way of treating a number of patients simultaneously.

Other group work, for example reminiscence sessions, have demonstrated that, for dementia patients, communication patterns can vary markedly between different linguistic contexts. One elderly lady, regarded as severely withdrawn and noncommunicative, surprised everyone when, in such a group, she asked for her favourate perfume of which she had just been reminded. Video recording of these groups has taken place with the intention of teaching skills to others. Also, so fundamental is the problem of communication breakdown that the speech therapist has quite naturally assumed a key role in running relative support groups.

Thus, following an accurate assessment to identify strengths and deficits of communication, we have found that an important speech therapy role is in identifying which linguistic and environmental factors can be manipulated to improve communication. This then becomes a strategy for carers and/or professional staff more so than attempting to teach the patient specific skills, although this too is necessary at times. Such plans need to be regularly updated in the light of the progressive nature of most of the disorders.

Although most referrals were for patients with dementia, nearly a quarter were for non-organically impaired patients: mainly individuals with more chronic forms of depression. Such individuals were seen while in-patients. They were typically withdrawn, rarely spontaneous in their speech, and had limited non-verbal communication as reflected in facial expression, physical gestures and posture. A recent study (Pentland et al, 1988) noted that senior speech therapy students made erroneous assumptions about the personality and intelligence of patients with reduced expressive behaviour (in this case due to Parkinson's disease) and rated them as less likeable than controls. Since factors such as this, which are just as likely with depressed patients, may adversely influence staff-patient interactions ward-based groups led by the speech therapist have been established to facilitate communication and minimise any institutionalising effects by encouraging choice and decision making. 


\section{Research}

As well as being essential to management, an accurate assessment of language skills is also the starting point for collecting information about the nature of linguistic breakdown within dementia. For a long time progress was held back by applying the label 'dysphasia' to any language difficulty within the dementias. It is now recognised that the specific deficits of dysphasia are different from the patterns of disruption in dementia where, for example, in Alzheimer's disease there is known to be interference with the higher order semantic aspects of language. However, existing research has often been hard to interpret because the instruments used have been devised for dysphasia research and have not always been appropriate for the study of dementia.

A pilot project was therefore conducted on all new attendances in a given period to the psychogeriatric day hospital using a visual confrontation naming task. Results indicated that the majority of patients' errors often were semantically related to the target, but visual misperception also appeared a possible explanation as this too was common. However, it became clear that existing scoring systems did not do justice to the range of errors which these patients with dementia produced. This has led to the development of a test which can score errors more discriminantly. Initial work with this test within the Department has strengthened the view that the majority of errors made by dementia patients are semantically related rather than simply visual misperceptions. The next task is to attempt to locate the level of disruption that this represents within the organisation of language and to what extent this differs between various aetiological groups. Since we have adjacent offices this has led to some useful exchanges of ideas along with helpful discussions with other disciplines within the Department such as psychology and occupational therapy. Often the difficulty in interpreting other studies has arisen because research has been conducted by staff isolated from the relevant clinical areas.

The effectiveness of various therapeutic interventions such as the running of communication groups in old people's homes and workshops for care assistants has been evaluated (Rising Tide Programme, 1987), as has the role of reminiscence in facilitating conversation with the elderly confused (Burford \& Griffiths, 1989). Guidelines for others running such groups have been developed and the skills have been passed on to other disciplines, whose own success may depend upon optimal communication with the patient.
It is interesting that referral patterns have indicated a perceived need for communication work with depressed patients. Since research is scant, HG now has an input into the Department's current depression study in order to explore this further: another opportunity made possible by being an established member of the multidisciplinary team.

\section{Teaching}

Not surprisingly, a considerable teaching commitment has developed: on a formal level to various student groups and qualified therapists and on an informal basis to other members of the multidisciplinary team, home helps, etc., including talks and demonstrations.

So great is the perceived need among speech therapists in understanding work within this field that a conference organised by HG was oversubscribed. Support and interest has also come from a North West interest group of speech therapists interested in the problems of language within dementia.

It is to be hoped that speech therapy as a discipline will develop further in the field of old age psychiatry, but it is already possible to identify specific areas where a therapist can make a significant contribution to the care of the elderly mentally ill. Clinicians working with the elderly need to be aware of this, especially when planning or reorganising services leads to an opportunity to secure funding for some speech therapy sessions.

\section{References}

BAYLES, K. A. (1982) Language function in senile dementia. Brain and Language, 16, 265-280.

- \& KoszniaK, A. (1987) Communication and Cognition in Normal Aging and Dementia. London: Taylor \& Francis.

BURFORD, A. \& GRIFFITHS, H. L. (1989) Reminiscence with the elderly mentally ill. Nursing Times (in press).

GilleARD, C. J. (1984) Living with Dementia. London: Croom Helm.

Neary, D., Snowden, J. S., Northen, B. \& Goulding, P. (1988) Dementia of the frontal type. Journal of Neurology, Neurosurgery and Psychiatry, 51, 353-361.

Pentland, B., Gray, J. M., Riddle, W. J. R. \& Pitcairn, T. K. (1988) The effects of reduced non-verbal communication in Parkinson's disease. British Journal of Disorders of Communication, 23, 31-34.

Rising TIDE PROGRAMME (1987) A report of progress made on the Central Manchester District Rising Tide Initiative 1983-1987; unpublished internal report. 